\title{
Is There a Role for Micronutrients in the Prevention of Cognitive Declines Associated with Aging?
}

\section{Mary Harris*}

Department of Food Science and Human Nutrition, Colorado State University, Fort Collins, USA

As life expectancy continues to increase, healthy aging will become one of the most important challenges of the $21^{\text {st }}$ century. In the new era of vitamin and micronutrient research, the study of nutrient requirements has shifted from an emphasis on prevention of deficiency to the promotion of optimum health and prevention of age related chronic disease. Some of the major biological changes from lifetime accumulation of molecular damage at the cellular level may be influenced by vitamin and micronutrient intakes and, certainly, most of the major health problems associated with aging are at least partially attributable to lifestyle and environmental factors. Thus, the potential impact of nutrition on the promotion of successful aging has become a topic of increasing interest. Declining cognitive ability, a major deterrent to successful aging, has recently been linked to diet and lifestyle factors. A growing body of knowledge is providing evidence that vitamins, trace minerals and lipids, can modify the risk of cognitive decline and dementia, especially in the frail elderly at risk of deficiencies. The focus has largely revolved around the B-vitamins involved in homocysteine metabolism (folate and B12), antioxidant nutrients (vitamins E, C and carotenoids), vitamin D and dietary lipids.

Although results of epidemiological studies are sometimes conflicting, certain associations have frequently been demonstrated. Diets rich in fruits and vegetables, omega-3 fatty acids, vitamins and trace elements are protective against cognitive decline, whereas diets high in saturated and trans- fatty acids are associated with increased risk of cognitive impairment in the elderly [1]. Numerous longitudinal cohort studies including the Zutphen Elderly Study, [2] the Atherosclerotic Risk in Communities Study [3] in the US and the EVA [4] study in France, link fish consumption with lower risk of cognitive decline. Fish is a rich source of omega- 3 docosahexaenoic acid (DHA) and eicosapentaenoic acid (EPA), vitamin D and selenium. The effect of fish consumption on cognitive performance may be related to the contribution of any, or a combination of, these nutrients. The intake of omega-3 DHA and EPA intake has been associated with decreased risk for self-reported cognitive difficulties [5], and Bourkje et al. [2] demonstrated a strong inverse association between intake of EPA+DHA and cognitive decline. However these results were not able to be substantiated in the US Veterans Administration Normative Aging Study [6] or in the earlier report from the Zutphen Elderly Study [7] or the Canadian Study of Health and Aging [8]. It has been suggested that cognitive function in the elderly declines differentially in specific cognitive domains, some more related to development of dementia than others [9]. Measurement of cognitive decline may be impacted by the measurement used. For example, omega-3 fatty acids may reverse negative effects of short term memory associated with stress or depression induced changes in the hypothalamic-pituitary adrenal axis and be ineffective in individuals with normal cortisol levels.

Epidemiologic data and long term supplementation trials suggest a protective role of the B-vitamins, especially folate and B12, on cognitive decline and dementia. The McArthur Study of Successful Aging in California [10] showed that, in high-functioning older adults, low folate levels appeared to be a risk factor for cognitive decline and that the risk of developing cognitive decline might be reduced by increasing dietary folate intake. An Australian randomized controlled supplementation trial with folate and B12 in a cohort of community- dwelling older adults given $400 \mu \mathrm{g}$ folate+ $100 \mu \mathrm{g}$ B-12 daily showed improved cognitive function scores (both immediate and delayed memory performance) after two years compared to placebo, but no significant changes in measures of orientation, attention, semantic memory or processing speed [11]. Recently, a meta-analysis [12] examined the effect of folic acid supplementation on the prevention of cognitive decline in nine placebo-controlled randomized trials (including 2835 subjects with a median study duration of six months) of folic acid supplementation on cognitive function and concluded that the trials show no effect of folic acid, with or without other B vitamins, on cognitive function.Trials of longer duration, evaluating cognitive declines as well as examining specific domains of cognitive loss associated with progression to dementia which are likely to be impacted by the folate/B12/homocysteine mechanism are needed.

Apart from the traditional role for vitamin D in calcium metabolism and bone health, the role for vitamin $\mathrm{D}$ in brain development and function has been gaining support over the last decade. Emerging evidence suggests that vitamin $\mathrm{D}$ acts as a neuroactive steroid to promote brain development and maintain brain function in adults [13]. Vitamin D deficiency in adults has been associated with development of Alzheimer's disease and cognitive decline. A limited number of studies evaluating serum vitamin D levels and cognitive impairment have yielded conflicting results, but in several studies, deficient levels of serum25-Hydroxyvitamin $\mathrm{D}(<25 \mu \mathrm{g} / \mathrm{dl})$ are associated with increased risk for cognitive impairment when adjusted for potential confounders [14]. Polymorphisms in the Vitamin D receptor (VDR) gene have been linked with gender-specific cognitive changes in the Baltimore Longitudinal Study of Aging study [15].

Current theories suggest that the protective effects of a diet rich in fruits and vegetables may be related to reversal of suboptimal antioxidant status. Inflammation and oxidation are important processes involved in the development of cognitive impairment and dementia. Antioxidant properties of some vitamins and trace elements may help to prevent cognitive decline. Reports of the potential effects of vitamin antioxidants in preventing or slowing the progression of dementia and cognitive decline are conflicting. In the Cache-County Study, high intakes of vitamins $\mathrm{C}$ and $\mathrm{E}$ and carotene were associated with lower risk of cognitive decline [16]. Long-term effects of antioxidant nutrient supplementation on cognitive performance were examined in the six year supplementation in the FrenchVitamins and Mineral Antioxidants (SU.VI.MAX) trial [17]. In this large randomized placebo-

*Corresponding author: Dr. Mary Harris, Professor, Department of Food Science and Human Nutrition, Colorado State University, Fort Collins, USA, Tel: 970-4917462; Fax: 970-491-7252; E-mail: mary.harris@colostate.edu

Received January 19, 2012; Accepted January 19, 2012; Published January 23 2012

Citation: Harris M (2012) Is There a Role for Micronutrients in the Prevention of Cognitive Declines Associated with Aging? Vitam Trace Elem 1:e105.

Copyright: (c) 2012 Eskin NAM. This is an open-access article distributed under the terms of the Creative Commons Attribution License, which permits unrestricted use, distribution, and reproduction in any medium, provided the original author and source are credited. 
controlled trial, participants received moderate daily doses of vitamin C, $\beta$-carotene, vitamin E, selenium and zinc or placebo. Subjects receiving antioxidant supplements had better episodic memory scores, although verbal memory was improved only in specific groups such as nonsmokers with low baseline serum vitamin C levels. Still other studies have failed to show protective effects of antioxidant vitamins on cognitive decline [6].

Emerging evidence suggests a possible protective effect of vitamins, trace minerals and omega- 3 fatty acids in relation to cognitive decline, but the benefits demonstrated in the current trials are modest and further evidence is required for confirmation. There is a need to develop more prospective studies of sufficient duration, in which diet is monitored before chronic conditions and cognitive declines occur, and data are collected to control for known confounding factors. Long term dose response, randomized clinical trials and meta-analyses of the most appropriate existing clinical trials need to be conducted to confirm the protective effect of vitamins and trace nutrients on cognitive function. Specific changes in cognitive domains associated with progression to dementia and other forms of cognitive impairment as well as global evaluations of cognitive function need to be considered to clarify the role of micronutrients in the prevention of cognitive declines associated with aging.

\section{References}

1. Bowman GL, Silbert LS, Howieson D, Dodge HH, Traber MG, et al. (2011) Nutrient biomarker patterns, cognitive function and MRI measures of brain aging. Neurology.

2. van Gelder BM, Tijhuis M, Kalmijn S and Kromhout D (2007) Fish consumption, n-3 fatty acids, and subsequent 5-y cognitive decline in elderly men: the Zutphen Elderly Study. Am J Clin Nutr 85: 1142-1147.

3. Beydoun MA, Kaufman JS, Satia JA, Rosamond W, Folsom AR (2007) Plasma n-3 fatty acids and the risk of cognitive decline in older adults: the Atherosclerosis Risk in Communities Study. Am J Clin Nutr 85: 1103-1111.

4. Heude B, Ducimetiere P and Berr C (2003) Cognitive decline and fatty acid composition of erythrocyte membranes-- the EVA Study. Am J Clin Nutr 277: 803-808.

5. Kesse-Guyot E, Péneau S, Ferry M, Jeandel C, Hercberg S, et al. (2011) Thirteen-year prospective study between fish consumption, long-chain n-3 fatty acids intakes and cognitive function. J Nutr Health Aging 15: 115-120.
6. van de Rest O, Spiro A, Krall-Kaye E, Geleijnse JM, de Groot, LC, et al. (2009) Intakes of (n-3) fatty acids and fatty fish are not associated with cognitive performance and 6-year cognitive change in men participating in the Veterans Affairs Normative Aging Study. J Nutr 139: 2329-2336.

7. Kalmijn S, Feskens EJ, Launer LJ, Kromohout D (1997) Polyunsaturated fatty acids, antioxidants, and cognitive function in very old men. Am J Epidemiol 145: 33-41.

8. Kröger E, Verreault R, Carmichael PH, Julien P, Dewailly E, et al. (2009) Omega-3 fatty acids and risk of dementia: the Canadian Study of Health and Aging. Am J Clin Nutr 90: 184-192.

9. Grodstein F, Chen J, Willett WC (2003) High-dose antioxidant supplements and cognitive function in community-dwelling elderly women. Am J Clin Nutr 77: $975-984$

10. Kado DM, Karlamangla AS, Huang MH, Troen A, Rowe JW, et al. (2005) Homocysteine versus the vitamins folate, $\mathrm{B} 6$, and $\mathrm{B} 12$ as predictors of cognitive function and decline in older high-functioning adults: MacArthur Studies of Successful Aging. Am J Med 118: 161-167.

11. Walker JG, Batterhan PJ, Mackinnon AJ, Jorm AF, Hickie I, et al. (2012) Ora folic acid and vitamin B-12 supplementation to prevent cognitive decline in community-dwelling older adults with depressive symptoms-the Beyond Ageing Project: a randomized controlled trial. Am J Clin Nutr 95: 194-203.

12. Wald DS, Kasturiratne A, Simmonds M (2010) Effect of folic acid, with or without other B vitamins, on cognitive decline: meta-analysis of randomized trials. Am J Med 123: 522-527.

13. Kesby JP, Eyles DW, Burne TH, McGrath JJ (2011) The effects of vitamin D on brain development and adult brain function. Mol Cell Endocrinol 347: 121-127.

14. Llewellyn DJ, Lang IA, Langa KM, Muniz-Terrera G, Phillips CL, et al. (2010) Vitamin D and risk of cognitive decline in elderly persons. Arch Intern Med170: 1135-1141.

15. Beydoun MA, Ding EL, Beydoun HA, Tanaka T, Ferrucci L, et al. (2012) Vitamin $D$ receptor and megalin gene polymorphisms and their associations with longitudinal cognitive change in US adults. Am J Clin Nutr 95: 163-178.

16. Wengreen HJ, Munger RG, Corcoran CD, Zandi P, Hayden KM, et al. (2007) Antioxidant intake and cognitive function of elderly men and women: the Cache County Study. J Nutr Health Aging 11: 230-237.

17. Kesse-Guyot E, Fezeu L, Jeandel C, Ferry M, Andreeva V, et al. (2011) French adults' cognitive performance after daily supplementation with antioxidan vitamins and minerals at nutritional doses: a post hoc analysis of the Supplementation in Vitamins and Mineral Antioxidants (SU.VI.MAX) trial. Am J Clin Nutr 94: 892-899. 